\title{
Endocytic mechanisms and osteoinductive profile of hydroxyapatite nanoparticles in human umbilical cord Wharton's jelly-derived mesenchymal stem cells
}

This article was published in the following Dove Press journal:

International Journal of Nanomedicine

\section{Xingxing Shi* \\ Kai Zhou* \\ Fei Huang* \\ Juan Zhang \\ Chen Wang}

Department of Prosthodontics, Jiangsu Key Laboratory of Oral Diseases, Affiliated Hospital of Stomatology, Nanjing Medical University, Nanjing, China

*These authors contributed equally to this work
Correspondence: Chen Wang Department of Prosthodontics, Jiangsu Key Laboratory of Oral Diseases, Affiliated Hospital of Stomatology, Nanjing Medical University, No I36, Han-zhong Road, Nanjing 210029, China Tel +86258503 | $83 \mid$

Email chwang9122@sina.com

\begin{abstract}
Background: As a potentially bioactive material, the widespread application of nanosized hydroxyapatite (nano-HAP) in the field of bone regeneration has increased the risk of human exposure. However, our understanding of the interaction between nano-HAP and stem cells implicated in bone repair remains incomplete.
\end{abstract}

Methods: Here, we characterized the adhesion and cellular internalization of HAP nanoparticles (HANPs) with different sizes (20 nm np20 and $80 \mathrm{~nm} \mathrm{np} 80)$ and highlighted the involved pathway in their uptake using human umbilical cord Wharton's jelly-derived mesenchymal stem cells ( $\mathrm{hWJ}$-MSCs). In addition, the effects of HANPs on cell viability, apoptosis response, osteogenic differentiation, and underlying related mechanisms were explored.

Results: It was shown that both types of HANPs readily adhered to the cellular membrane and were transported into the cells compared to micro-sized HAP particles (m-HAP; $12 \mu \mathrm{m}$ ). Interestingly, the endocytic routes of np20 and np80 differed, although they exhibited similar kinetics of adhesion and uptake. Our study revealed involvement of clathrin- and caveolin-mediated endocytosis as well as macropinocytosis in the np20 uptake. However, for np80, clathrin-mediated endocytosis and some as-yet-unidentified important uptake routes play central roles in their internalization. HANPs displayed a higher preference to accumulate in the cytoplasm compared to m-HAP, and HANPs were not detected in the nucleolus. Exposure to np20 for $24 \mathrm{~h}$ caused a decrease in cell viability, while cells completely recovered with an exposure time of $72 \mathrm{~h}$. Furthermore, HANPs did not influence apoptosis and necrosis of hWJ-MSCs. Strikingly, HANPs enhanced mRNA levels of osteoblast-related genes and stimulated calcium mineral deposition, and this directly correlated with the activation in c-Jun N-terminal kinases and p38 pathways.

Conclusion: Our data provide additional insight about the interactions of HANPs with MSCs and suggest their application potential in hard tissue regeneration.

Keywords: HANPs, hWJ-MSCs, internalization, osteogenic differentiation, JNK, p38

\section{Introduction}

With the rapid advancement in nanotechnology, nanomaterials (NMs) possess unique physical, chemical, and biological properties and have thus attracted particular attention for various biomedical applications. Therefore, the interaction of biological systems with NMs has become an intriguing research area at the interface between biology and applied research. ${ }^{1,2}$ Nanosized hydroxyapatite (nano-HAP) is an important inorganic constituent of the native bone matrix and has been extensively investigated as a candidate for hard tissue regeneration and implants such as filling materials, scaffolds, 
and bioactive coatings. ${ }^{3-5}$ Increasing evidence suggests that, in addition to the ameliorated mechanical properties, ${ }^{6}$ nano-HAP is capable of promoting osteoblast-like cells proliferation in vitro and improving bone repair in vivo..$^{7-9}$ Nevertheless, the potential health effects that may be elicited by nano-HAP (especially in bone defect sites) remain yet to be elucidated. For the clinical application of nano-HAP, a more comprehensive understanding of the induced biological responses is warranted.

Bone regeneration is a physiological event, which involves complex and diverse cellular actions, such as migration and differentiation of mesenchymal stem cells (MSCs). As artificial bone materials, when nano-HAP is implanted into the body, it might be taken up by cells due to its comparable length scale to proteins and discrete components of cells. Consequently, it may exert effects on cell function, thus influencing bone repair. MSCs are characterized by their potential for self-renewal as well as by multi-differentiation and play an essential role in regenerative and reparative medicine. It is imperative to determine the effects of bone substitutes on fundamental biological functions of MSCs. To understand how nano-HAP could influence MSCs and to obtain information about its biocompatibility, understanding the mechanisms that underlie internalization and the intracellular fate of nano-HAP is of paramount importance. It has been reported that osteoblastic cells could endocytose HAP nanoparticles (HANPs $)^{10}$ and as delivery vehicle, HANPs can be taken up by liver cancer cells through clathrin-mediated endocytosis. ${ }^{11}$ However, so far, the internalization of HANPs in MSCs, especially the route of uptake that might mediate such a process, remains unknown. On the other hand, although some studies have indicated that nano-HAP is implicated in the modulation of osteogenic differentiation of MSCs, ${ }^{12,13}$ the precise mechanisms remain unknown.

In this study, we explored detailed uptake kinetics and internalization pathways of HANPs of different size in human umbilical cord Wharton's jelly-derived MSCs (hWJ-MSCs). To gain further insight into the effect of HANPs on biological functions of MSCs, a number of assessment techniques, including cell viability, apoptosis response, cell cycle, and osteogenic differentiation, were applied. Furthermore, pathways of osteoinductivity of HANPs were also investigated to determine intrinsic mechanisms.

\section{Materials and methods Particles and their characteristics}

HANPs ( $20 \mathrm{~nm}$ np20 and $80 \mathrm{~nm}$ np 80 ) and micro-sized HAP particles (m-HAP, $12 \mu \mathrm{m}$ ) were purchased from Nanjing
Emperor Nano Material Company Ltd (Nanjing, China). The particles were viewed using transmission electron microscopy (TEM, FEI Tecnai G2 Spirit BioTwin; FEI, Hillsboro, OR, USA) and scanning electron microscopy (SEM, LEO1530VP, Germany). The hydrodynamic size and zeta potential of HAP particles (HAPs) in low-glucose Dulbecco's Modified Eagle's Medium (L-DMEM, Gibco-Invitrogen Co, Waltham, MA, USA) containing 10\% fetal bovine serum (FBS, GibcoInvitrogen Co) were determined via Zetasizer Nano ZS90 and Mastersizer 3000 (Malvern Instruments, Malvern, UK). HAPs were sterilized with epoxyethane.

To explore cellular uptake, fluorescein isothiocyanate (FITC) was conjugated to the surface of HAPs. Briefly, $0.05 \mathrm{~g}$ of HAPs was mixed in $50 \mathrm{~mL}$ anhydrous ethanol, then $10 \mathrm{~mL}$ 3-aminopropyltriethoxysilane (Sigma, St Louis, MO, USA) was dispersed, and the temperature was maintained at $74^{\circ} \mathrm{C}$ under constant stirring. After $3 \mathrm{~h}, 0.025 \mathrm{~g}$ FITC was added to the solution and reaction was conducted for $6 \mathrm{~h}$. The treated HAPs were filtered prior to washing with anhydrous ethanol followed by ultrapurified water. FITC-labeled HAPs (FITC-HAPs) were obtained via lyophilizing.

\section{Cell preparation and culture}

All experimental protocols were approved by the ethics committee of Nanjing Medical University. hWJ-MSCs were isolated from fresh human umbilical cords as previously described after obtaining written informed consent from the donors. ${ }^{14}$ Briefly, the umbilical cord was washed thrice with phosphate-buffered saline (PBS), containing $100 \mu \mathrm{g} / \mathrm{mL}$ penicillin and streptomycin $\left(\right.$ Gibco $^{\circledR}$; Thermo Fisher Scientific, Waltham, MA, USA). After removal of blood vessels, the Wharton's jelly was cut into $1 \mathrm{~mm}^{2}$ pieces, then placed in $25 \mathrm{~cm}^{2}$ tissue culture flasks (Corning Incorporated, Corning, NY, USA), and incubated in L-DMEM supplemented with $10 \% \mathrm{FBS}, 100 \mathrm{U} / \mathrm{mL}$ penicillin, and $100 \mu \mathrm{g} / \mathrm{mL}$ streptomycin. Non-adherent Wharton's jelly pieces were removed after 3 days. Cell surface marker analysis was performed to confirm the phenotype of hWJ-MSCs, using monoclonal antibodies for CD13, CD29, CD34, CD44, CD45, CD51, and CD105 (BD Biosciences, San Jose, CA, USA).

\section{Cell viability assay}

Cell viability was measured by 3-[4,5-dimethylthiazol-2yl]-5-[3-carboxymethoxyphenyl]-2-[4-sulfophenyl]-2Htetrazolium, inner salt (MTS, Bestbio, Shanghai, China) colorimetric assay. Briefly, hWJ-MSCs at a density of $5 \times 10^{3}$ per well were seeded in 96-well culture plates. Then, media containing HAPs $(10,25,50,100$, and $200 \mu \mathrm{g} / \mathrm{mL})$ 
were added and cultured for 24 and $72 \mathrm{~h}$, respectively. The assay was performed in accordance with manufacturer's instructions. The optical density at $490 \mathrm{~nm}$ was quantitated using a microplate reader (SpectraMax M2, Molecular Devices LLC, Sunnyvale, CA, USA).

\section{Assessment of cell cycle and apoptosis}

For cell cycle analysis, hWJ-MSCs were harvested after HAPs treatment for $24 \mathrm{~h}$, and then washed with PBS. Subsequently, hWJ-MSCs were allowed to be fixed in $75 \%$ cold ethanol at $-20^{\circ} \mathrm{C}$ for $24 \mathrm{~h}$, and washed with PBS before the cells were stained with propidium iodide (PI) in RNase for $30 \mathrm{~min}$ at $37^{\circ} \mathrm{C}$. Then, all samples were analyzed using flow cytometry (FACSCalibur, BD Biosciences).

The percentages of apoptotic and necrotic cells were identified using the Annexin V-PI apoptosis detection kit (BD Biosciences). Briefly, hWJ-MSCs treated with serial dilutions of HAPs were collected after $24 \mathrm{~h}$ and washed with PBS. Samples were stained with Annexin V-FITC and PI as recommended by the manufacturer before the cells were assessed via flow cytometer.

\section{Uptake activity via TEM and confocal laser scanning microscopy (CLSM)}

To visualize cellular uptake, hWJ-MSCs were seeded in 6-well plates. After 1, 2, and $4 \mathrm{~h}$ of exposure to $25 \mu \mathrm{g} / \mathrm{mL}$ HANPs, cells were harvested and fixed with $2.5 \%$ glutaraldehyde. The uptake and intracellular localization of HANPs were then observed by TEM.

For CLSM imaging, hWJ-MSCs were plated on dedicated coverslips and exposed to FITC-HAPs at a dose of $25 \mu \mathrm{g} / \mathrm{mL}$ for 1,2 , and $4 \mathrm{~h}$, respectively. The cells were then fixed with $4 \%$ paraformaldehyde and then incubated with rhodaminephalloidin and DAPI. Images were taken using CLSM (LSM710, Carl Zeiss Meditec AG, Jena, Germany).

\section{Kinetics of HAP adhesion and uptake}

To quantitatively characterize the adhesion properties of HAPs to hWJ-MSCs, flow cytometry analysis was performed according to previously published methodology. ${ }^{15}$ Briefly, hWJ-MSCs in 6-well plates were cultured with $25 \mu \mathrm{g} / \mathrm{mL}$ FITC-HAPs for indicated times at $4{ }^{\circ} \mathrm{C}$. After rinsing twice with PBS, cells were incubated in HAPs-free medium for $3 \mathrm{~h}$ at $37^{\circ} \mathrm{C}$ prior to examination via flow cytometry.

For determination of the uptake activity of HAPs by hWJ-MSCs, cells were seeded in 6-well plates and stimulated by $25 \mu \mathrm{g} / \mathrm{mL}$ FITC-HAPs for a range of different times at $37^{\circ} \mathrm{C}$. After few washing cycles, the samples were fixed in $1 \%$ paraformaldehyde. The cell fluorescence of HAPs was then analyzed using flow cytometry.

\section{Uptake mechanisms for HAPs in hWJ-MSCs}

For the study of uptake routes, three endocytosis inhibitors were applied. ${ }^{16,17}$ Chlorpromazine (cpz) was used to inhibit clathrin-mediated endocytosis. Inhibitions of clathrin- as well as caveolin-mediated endocytosis in hWJ-MSCs were tested via methyl- $\beta$-cyclodextrin $(\mathrm{m} \beta \mathrm{cd})$. Cytochalasin D (cytoD) specifically inhibits actin-dependent phagocytosis and macropinocytosis. Briefly, cells were exposed to $25 \mu \mathrm{g} / \mathrm{mL}$ of FITC-HAPs for $2 \mathrm{~h}$ after $30 \mathrm{~min}$ of pre-incubation with endocytotic inhibitors. Subsequently, images were taken using CLSM and cell fluorescence was quantified via flow cytometry.

\section{Alkaline phosphatase (ALP) staining}

ALP staining was performed with a commercially available kit (Beyotime, Shanghai, China). Briefly, hWJ-MSCs were seeded in 6-well plates and treated with different HAPs for 7 days in L-DMEM. Then, cells were fixed in 4\% paraformaldehyde and stained in a mixture of nitro-blue tetrazolium and 5-bromo-4-chloro-3-indolyl-phosphate. ${ }^{18}$

\section{Alizarin Red S staining}

The extracellular matrix calcification was observed using Alizarin Red S staining as previously described. ${ }^{19}$ In brief, hWJ-MSCs in 12-well plates were cultured in osteogenic medium (L-DMEM supplemented with 10\% FBS, $50 \mathrm{mg} / \mathrm{mL}$ L-ascorbic acid, $10 \mathrm{mM}$ glycerophosphate, and $100 \mathrm{nM}$ dexamethasone, Sigma) supplemented with HAPs for up to 14 days. After washing with PBS, the samples were fixed with $4 \%$ paraformaldehyde for $15 \mathrm{~min}$ and then stained in $1 \%(\mathrm{w} / \mathrm{v})$ Alizarin Red S ( $\mathrm{pH}, 4.1-4.5)$ at room temperature for $20 \mathrm{~min}$.

\section{Real-time polymerase chain reaction (PCR)}

Total RNA was isolated from hWJ-MSCs exposed to HAPs for 7,14 , and 21 days, respectively, using the TRIZOL reagent (Invitrogen Life Technologies, Carlsbad, CA, USA). Complementary DNA (cDNA) was synthesized from $1.0 \mu \mathrm{g}$ RNA using PrimeScript 1st Strand cDNA Synthesis kit (TaKaRa, Tokyo, Japan) in a T3 thermocycler (Mastercycler 5333, Eppendorf, Hamburg, Germany). Quantitative PCR was performed via the quantitative real-time amplification system (7900HT Fast, Applied Biosystems, Foster City, 
Table I Primers used for real time polymerase chain reaction

\begin{tabular}{|c|c|c|}
\hline Target gene & Forward primer sequence $\left(5^{\prime}-3^{\prime}\right)$ & Reverse primer sequence $\left(5^{\prime}-3^{\prime}\right)$ \\
\hline Runx-2 & CAGATGGGACTGTGGTTACTGT & GTGAAGACGGTTATGGTCAAGG \\
\hline Coll & АTCTCTCGAGCCCCAGCCACAAAGAGTCTA & TTCGCTCGAGACGACCAGGTTTTCCAGCTT \\
\hline$A L P$ & CCTGTGCATTTCCCAACACGGCGG & CCACTTCCCAGGCAGTGGAGACAG \\
\hline OCN & GGCAGCCTCTGATTGTGTCC & TATATCCACTGCCTGAGCGG \\
\hline GAPDH & GAAGGTGAAGGTCGGAGTC & GAGATGGTGATGGGATTTC \\
\hline
\end{tabular}

Abbreviation: $A L P$, alkaline phosphatase.

CA, USA) using the FastStart universal SYBR Green Master (ROX) kit (Roche, Basel, Switzerland). The housekeeping gene GAPDH was used as internal control, while the expression levels for each gene were normalized to that of GAPDH. The primer sequences for the selected genes are listed in Table 1.

\section{Western blotting}

For the apoptotic signaling pathway, hWJ-MSCs were incubated with HAPs for $24 \mathrm{~h}$, and lysed using ice-cold lysis buffer (50 mM Tris-HCl, $150 \mathrm{mM} \mathrm{NaCl}, 1 \% \mathrm{NP}-40$, $1 \%$ sodium deoxycholate, and $0.1 \%$ sodium dodecyl sulfate, Applygen, Beijing, China) for 30 min. Proteins from cell lysates $(40 \mu \mathrm{g})$ were separated on sodium dodecyl sulfate-polyacrylamide gel electrophoresis and transferred onto nitrocellulose membranes. After blocking with nonfat milk, the membranes were probed with anti-p-Akt, Akt, GAPDH (1:1,000, rabbit polyclonal antibodies, CST, Boston, MA, USA), anti-eNOS (1:500, a rabbit polyclonal antibody, CST), and anti-p-eNOS (1:1,000, a rabbit monoclonal antibody, CST) overnight at $4{ }^{\circ} \mathrm{C}$. Thereafter, the membranes were rinsed with Tris-buffered saline (TBS), containing $0.05 \%$ Tween-20 (TBST), followed by incubation with horseradish peroxidase-conjugated secondary antibodies at room temperature for $1 \mathrm{~h}$. The immunoreactive bands were measured via a chemiluminescence gel imaging system (LAS4000M, GE Healthcare Bio-Sciences AB, Uppsala, Sweden).

\section{Statistical analysis}

All data are expressed as mean \pm standard deviation of three separate experiments. Statistically significant differences $(P<0.05)$ of means were determined via one-way ANOVA. All statistical analyses were conducted using SPSS 18.0 software.

\section{Results \\ HAPs characterization}

HAPs were characterized for their particle shape, zeta potential, and hydrodynamic size. TEM and SEM images showed that np20 and m-HAP were near-spherical particles and about $20 \mathrm{~nm}$ and $12 \mu \mathrm{m}$ in diameter, respectively (Figure 1A and C). Np80 particles were rod-like, with approximate lengths of $80 \mathrm{~nm}$ and widths of $20 \mathrm{~nm}$ (Figure 1B). The zeta potential showed that the HAPs had negative surface charge in L-DMEM (Table 2). The hydrodynamic sizes of np20, np80, and m-HAP were $162.3 \mathrm{~nm}, 198.9 \mathrm{~nm}$, and $137 \mu \mathrm{m}$, respectively.

\section{Effect of HAPs on cell viability and cell cycle}

Cell metabolic activity was determined after exposing hWJ-MSCs to HAPs for 24 and $72 \mathrm{~h}$. As shown in Figure 2A, at low concentrations $(25 \mu \mathrm{g} / \mathrm{mL} \mathrm{np} 20$ and $50 \mu \mathrm{g} / \mathrm{mL}$ $\mathrm{np} 80$ ), promotion was observed for $\mathrm{np} 20$ and $\mathrm{np} 80$ for $24 \mathrm{~h}(P<0.05)$, while at the higher concentration of 100 and $200 \mu \mathrm{g} / \mathrm{mL}, \mathrm{np} 20$ demonstrated mild inhibition. However,
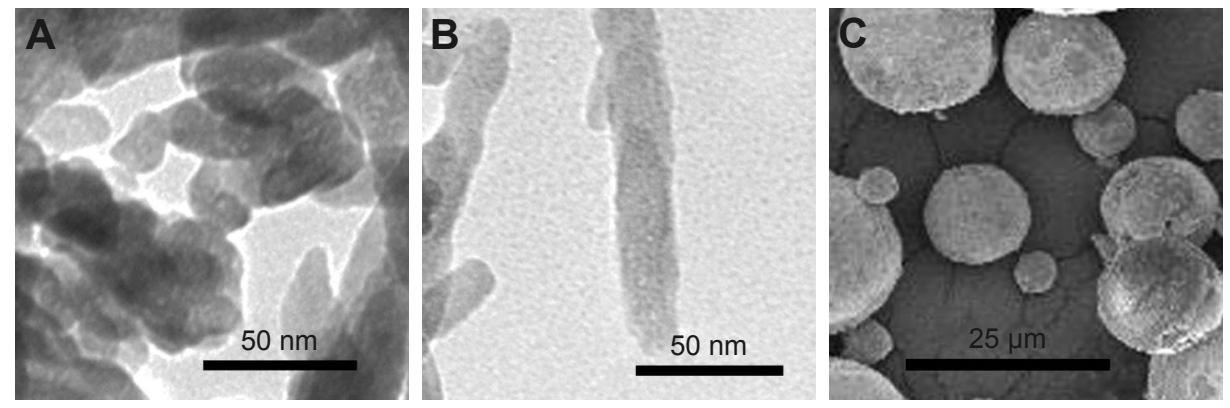

Figure I TEM micrographs of np20 (A) and np80 (B), and SEM micrograph of m-HAP (C).

Abbreviations: TEM, transmission electron microscopy; np20, hydroxyapatite nanoparticles $20 \mathrm{~nm}$ in diameter; np 80 , hydroxyapatite nanoparticles $80 \mathrm{~nm}$ in diameter; SEM, scanning electron microscopy; m-HAP, micro-sized HAP particles; HAP, hydroxyapatite. 
Table 2 Characterization of the particle parameters of HAPs

\begin{tabular}{lll}
\hline Material & $\begin{array}{l}\text { Hydrodynamic diameter } \\
\text { in L-DMEM with I0\% } \\
\text { FBS (average value) }\end{array}$ & $\begin{array}{l}\text { Zeta-potential } \\
\text { in L-DMEM with } \\
\text { I0\% FBS }(\mathbf{m V})\end{array}$ \\
\hline $\mathrm{np} 20$ & $162.3 \mathrm{~nm}$ & -9.13 \\
$\mathrm{np} 80$ & $198.9 \mathrm{~nm}$ & -9.68 \\
$\mathrm{~m}-\mathrm{HAP}$ & $137 \mu \mathrm{m}$ & -9.74 \\
\hline
\end{tabular}

Abbreviations: HAPs, hydroxyapatite particles; L-DMEM, low-glucose Dulbecco's Modified Eagle's Medium; FBS, fetal bovine serum; np20, hydroxyapatite nanoparticles $20 \mathrm{~nm}$ in diameter; np80, hydroxyapatite nanoparticles $80 \mathrm{~nm}$ in diameter; m-HAP, micro-sized HAP particles; HAP, hydroxyapatite.

following $72 \mathrm{~h}$ in culture with HANPs, cell viability did not show any significant change. Compared with HANPs and control, m-HAP induced dose-dependent reduction in cell viability; at the highest dose $(200 \mu \mathrm{g} / \mathrm{mL})$, cell viability decreased to approximately $50 \%$.

To understand the influence of HAPs on cell growth, the cell cycle was assessed. In the presence of np20 and m-HAP, no significant difference of cell cycle was detected compared to control $(P>0.05)$, while for the np80 group, the cell population in both $\mathrm{G} 2$ and $\mathrm{S}$ phases increased slightly (Figure 2B1-B3).

\section{Apoptosis and necrosis triggered by HAPs}

To further investigate the cytotoxicity of HAPs on hWJMSCs, apoptosis and necrosis were quantitatively estimated.
After $24 \mathrm{~h}$ of incubation with varying concentrations of HAPs, no toxic effects were detected regardless of size (Figure 3).

\section{Uptake of HAPs by hWJ-MSCs}

Cellular uptake is an important issue that indicates the biological response nanoparticles (NPs) may exert. Upon exposure to either HANPs or FITC-HAPNs at $25 \mu \mathrm{g} / \mathrm{mL}$ for 1,2 , and $4 \mathrm{~h}$, the uptake activities of HANPs by hWJ-MSCs were observed via TEM and CLSM. As shown in Figures 4 and 5, first HANPs adhered to cell membrane; then, cells stretched out pseudopodia to wrap them; and finally, HANPs were trafficked to the cytoplasm. Most of the HANPs were confined to lysosomes and vesicles of hWJ-MSCs, and did not enter the nucleolus. Along with the extension of time, HANPs accumulation was detected. By contrast, only a small amount of m-HAP was taken up during the observation. Moreover, exposure to HANPs and m-HAP had no appreciable effects on cellular morphology and structures of cell organelles in hWJ-MSCs.

\section{Kinetics of HAPs adhesion and uptake}

To conduct quantitative analysis for HAPs adhesion and uptake, their kinetics was assessed. As shown in Figure 6A,
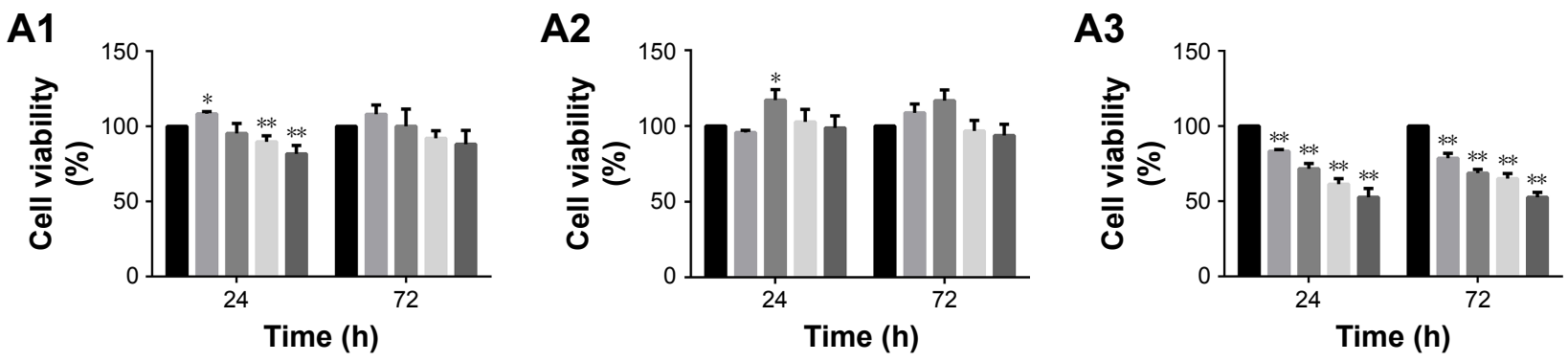

Control $25 \mu \mathrm{g} / \mathrm{mL} \quad 50 \mu \mathrm{g} / \mathrm{mL} \quad 100 \mu \mathrm{g} / \mathrm{mL} \quad 200 \mu \mathrm{g} / \mathrm{mL}$
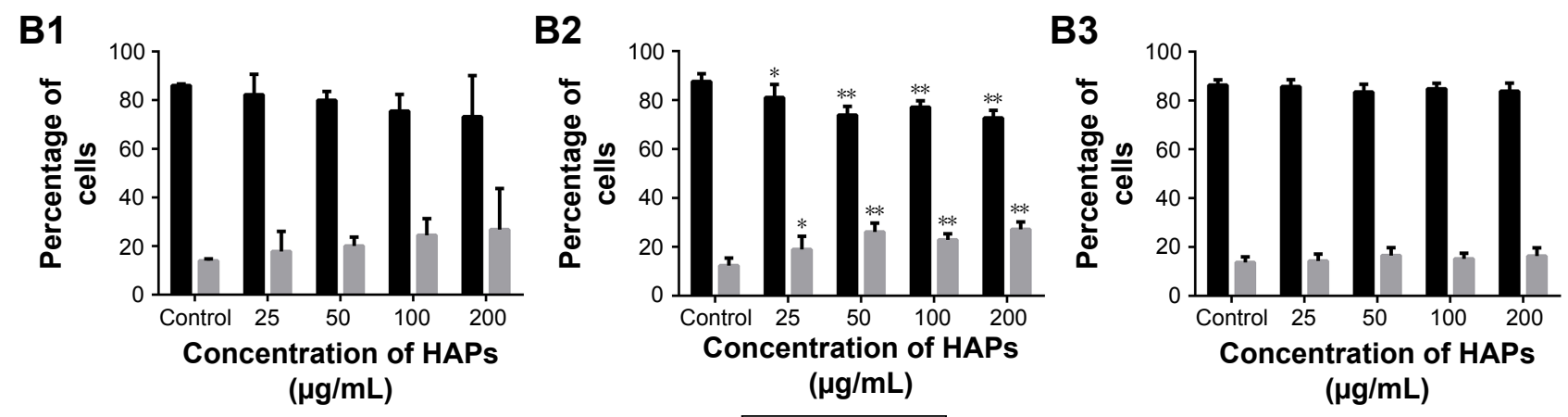

Figure 2 Effects of HAPs on cell viability and cycle of hWJ-MSCs. The viability of hWJ-MSCs cultured with np20 (AI), np80 (A2), and m-HAP (A3) for 24 and 72 h. Cell cycle of hWJ-MSCs after treatment with np20 (BI), np80 (B2), and m-HAP (B3) for $24 \mathrm{~h}$. $* \mathrm{P}<0.05$; $* * P<0.0 \mathrm{I}$ versus control. Cells without HAPs treatment were set as the control group.

Abbreviations: HAPs, hydroxyapatite particles; hWJ-MSCs, human umbilical cord Wharton's jelly-derived mesenchymal stem cells; np20, hydroxyapatite nanoparticles $20 \mathrm{~nm}$ in diameter; np80, hydroxyapatite nanoparticles $80 \mathrm{~nm}$ in diameter; m-HAP, micro-sized HAP particles; HAP, hydroxyapatite. 
A

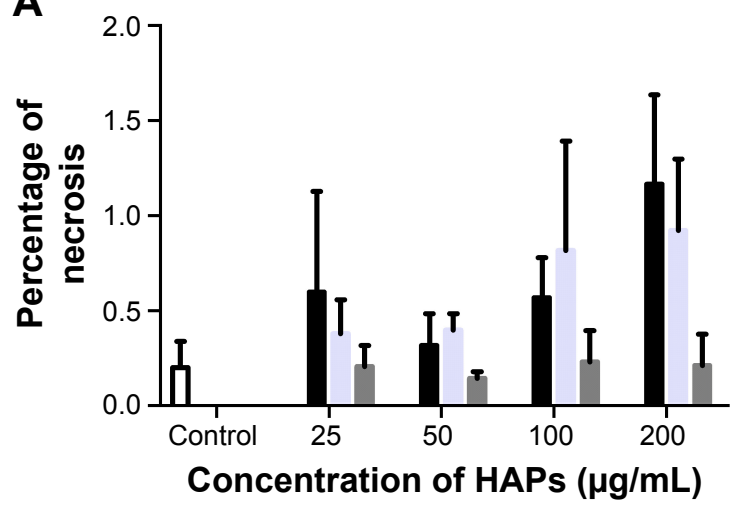

B

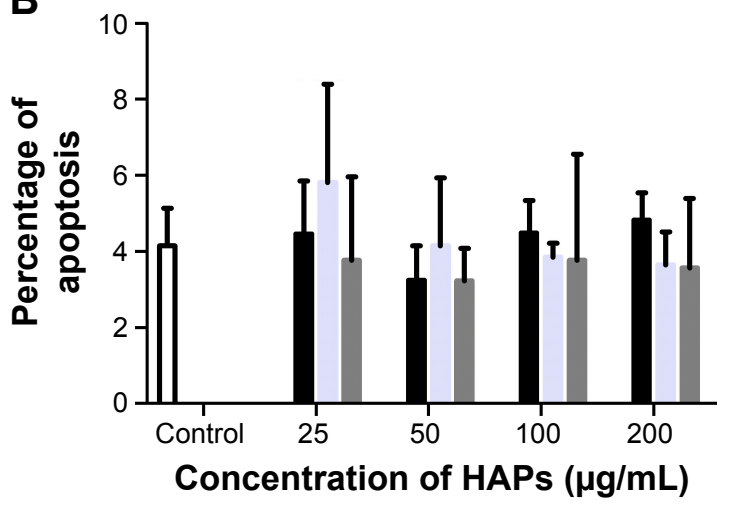

Figure 3 A subpopulation of necrotic (A) and apoptotic cells (B) following HAPs treatment for 24 h. Cells without HAPs treatment were set as the control group. Abbreviations: HAPs, hydroxyapatite particles; np20, hydroxyapatite nanoparticles $20 \mathrm{~nm}$ in diameter; np 80 , hydroxyapatite nanoparticles $80 \mathrm{~nm}$ in diameter; m-HAP, micro-sized HAP particles; HAP, hydroxyapatite.

the adhesion of HANPs to the hWJ-MSCs was a two-step process. Rapid growth in the adsorbed amount happened within the first $10 \mathrm{~min}$, after that, it reached a dynamic equilibrium at the time of trial runs. This trend also held true for m-HAP, whereas cell fluorescence of m-HAP was much lower than that of HANPs. There was no significant difference in the adsorbed amount between the np20 group and the np 80 group.
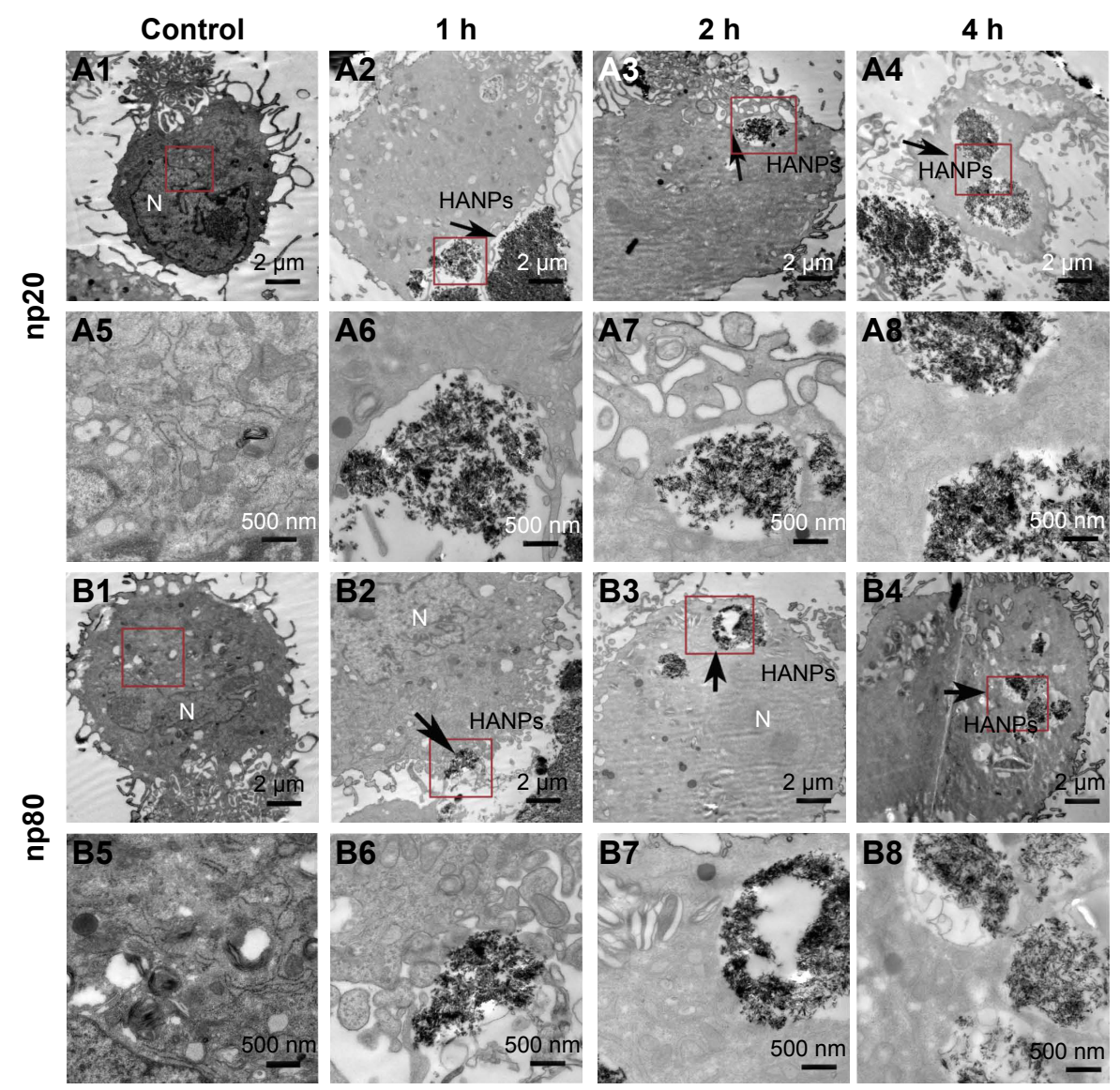

Figure 4 TEM micrographs of internalization and intracellular distribution of HANPs in hWJ-MSCs exposed for I, 2, and 4 h. hWJ-MSCs without any treatment (AI, A5, B I, and B5); hWJ-MSCs treated with np20 (A2-A4 and A6-A8) and np80 (B2-B4 and B6-B8). Overall cell morphology (AI-A4 and BI-B4), scale bars: 2 $\mu$ m. Higher magnification of cells in red-boxed areas (A5-A8 and B5-B8), scale bars: $500 \mathrm{~nm}$.

Abbreviations: TEM, transmission electron microscopy; HANPs, hydroxyapatite nanoparticles; hWJ-MSCs, human umbilical cord Wharton's jelly-derived mesenchymal stem cells; np20, hydroxyapatite nanoparticles $20 \mathrm{~nm}$ in diameter; np80, hydroxyapatite nanoparticles $80 \mathrm{~nm}$ in diameter; $\mathrm{N}$, cell nucleus. 


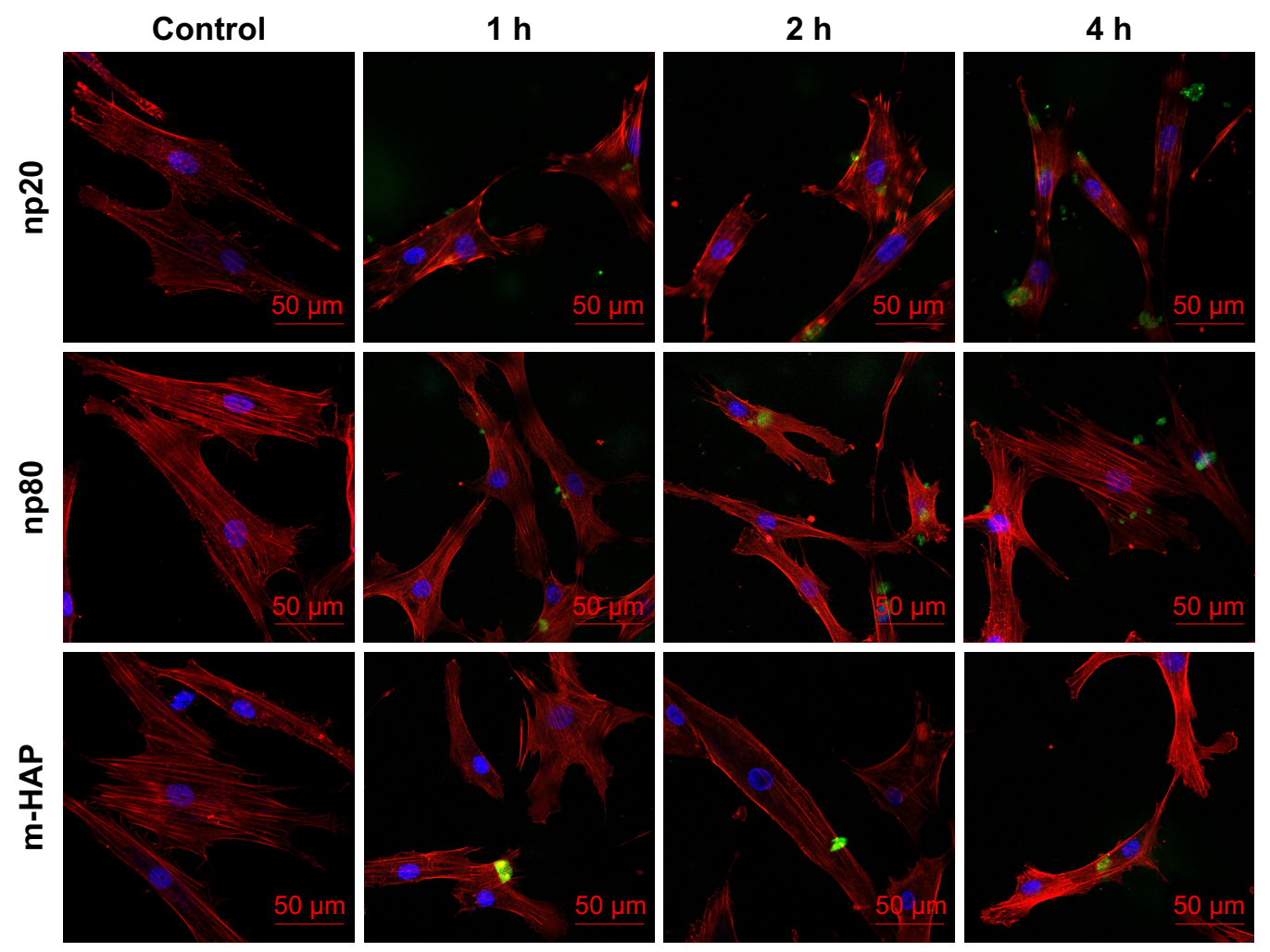

Figure 5 CLSM micrographs of internalization and subcellular distribution of HAPs in hWJ-MSCs exposed for I, 2, and 4 h. Cells stained for nuclei (blue) and actin (red). HAPs are shown in green color. Scale bars: $50 \mu \mathrm{m}$.

Abbreviations: CLSM, confocal laser scanning microscopy; HAPs, hydroxyapatite particles; hWJ-MSCs, human umbilical cord Wharton's jelly-derived mesenchymal stem cells; np20, hydroxyapatite nanoparticles $20 \mathrm{~nm}$ in diameter; np80, hydroxyapatite nanoparticles $80 \mathrm{~nm}$ in diameter; m-HAP, micro-sized HAP particles; HAP, hydroxyapatite.

The uptake kinetics of HAPs by hWJ-MSCs is indicated in Figure 6B; the cell fluorescence signal showed an initial transient, increasing linearly with time during the observation. Both the initial cell fluorescence and their growth rate were very close in the np 20 and np 80 groups followed by a rapid increase with time. After $60 \mathrm{~min}$, a relatively steady state had been reached. In comparison to np20, the uptake speed of np80 was slower; however, the fluorescence was higher when the equilibrium was reached. Clearly, the lower initial fluorescence of m-HAP resulted in a smaller amount that was taken up compared to HANPs, although it grew almost as quickly as HANPs.

\section{The activation of internalization pathways in $\mathrm{hWJ}$-MSCs by HAPs}

The observation that the HANPs could be taken up by hWJMSCs has led to question the endocytic pathways that were
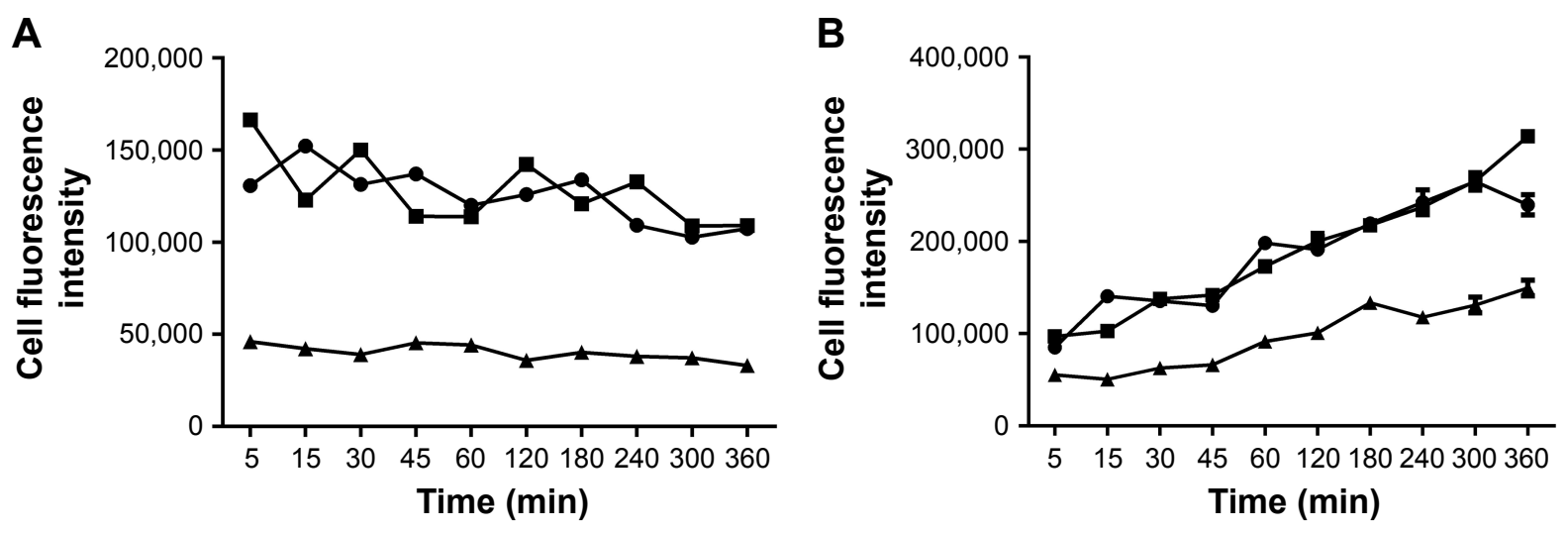

$$
\rightarrow \mathrm{np20} \rightarrow \mathrm{np80} \leftarrow \mathrm{m}-\mathrm{HAP}
$$

Figure 6 Kinetics of adhesion of HAPs onto hWJ-MSCs (A). Uptake kinetics of HAPs by hWJ-MSCs (B).

Abbreviations: HAPs, hydroxyapatite particles; hWJ-MSCs, human umbilical cord Wharton's jelly-derived mesenchymal stem cells; np20, hydroxyapatite nanoparticles $20 \mathrm{~nm}$ in diameter; np80, hydroxyapatite nanoparticles $80 \mathrm{~nm}$ in diameter; m-HAP, micro-sized HAP particles; HAP, hydroxyapatite. 
involved in these processes. Thus, cellular uptake variations in hWJ-MSCs that were exposed to different endocytosis inhibitors were quantified via CLSM and flow cytometry. The results have been summarized in Figure 7. The addition of cpz slightly decreased cell fluorescence without a significant difference among the three groups. Comparatively, treatment of hWJ-MSCs with $\mathrm{m} \beta \mathrm{cd}$ also resulted in a decreased uptake of HAPs. In this case, cell fluorescence in np20, np80, and m-HAP significantly decreased to $50.53 \%, 73.16 \%$, and $90.29 \%$, respectively. For cytoD, a dramatic drop in cell fluorescence was also observed in np20 and m-HAP groups (by about $40 \%$ and $50 \%$, respectively, compared to samples without inhibitor), whereas the reduction in np80 was unremarkable compared to samples without inhibitor.

\section{Osteogenic differentiation of hWJ-MSCs cultured with HAPs}

To reveal whether HANPs could affect the osteogenic differentiation of hWJ-MSCs, ALP staining and Alizarin Red S staining were performed. As shown in Figure 8A, no significant difference in ALP expression was detected among the three groups on day 7; however, more mineralized matrix depositions of hWJ-MSCs were observed in HANPs compared with m-HAP and control (Figure 8B).
A
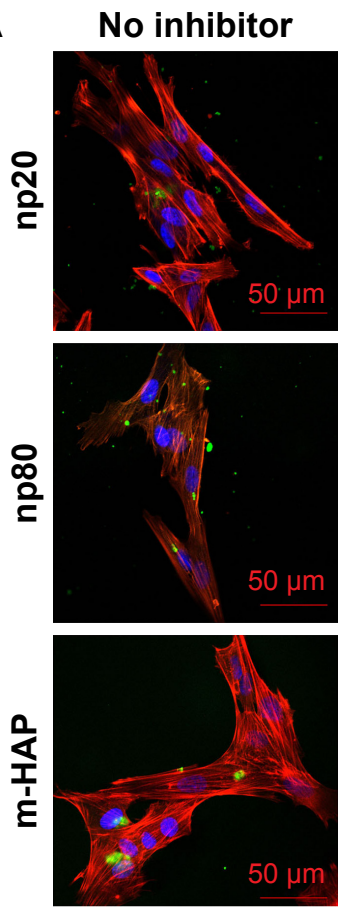

$\mathrm{cpz}$
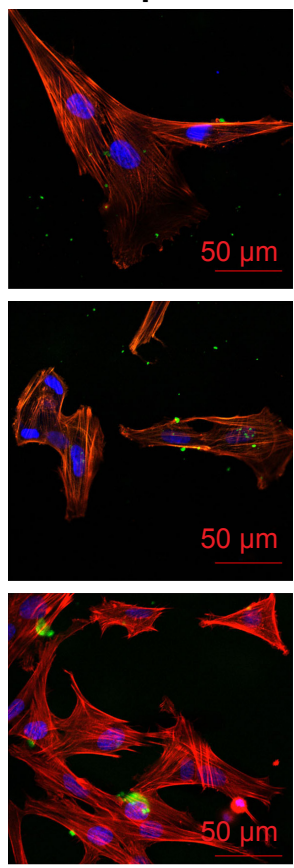

$\mathrm{m} \beta \mathrm{cd}$
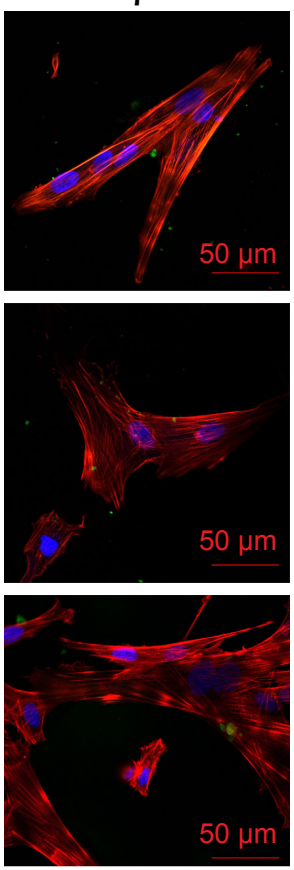

cytoD
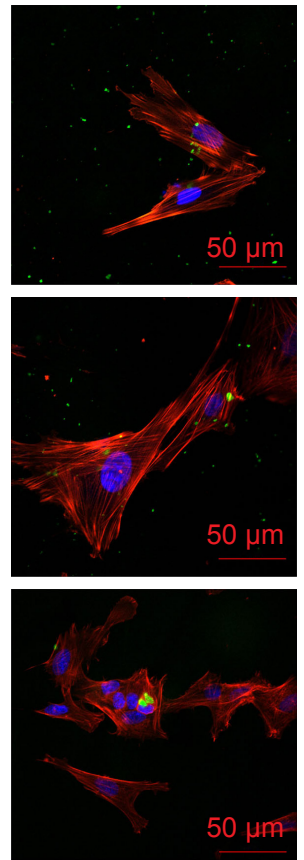

B

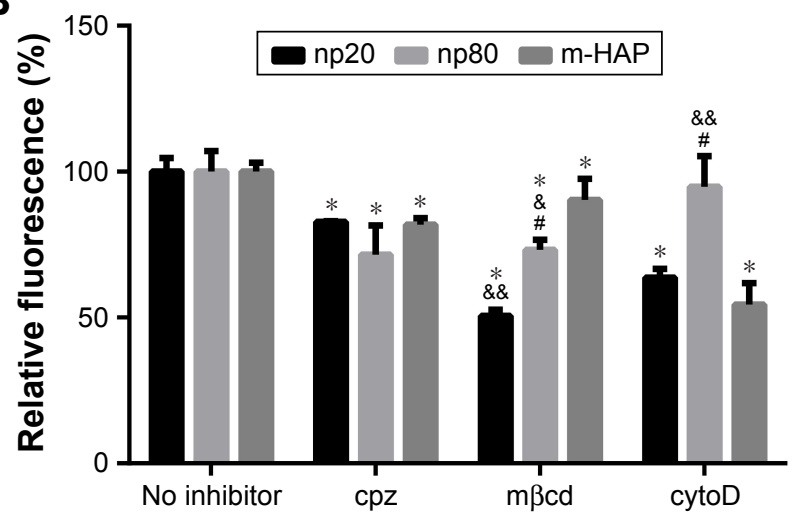

Figure 7 The role of different endocytic pathways in HAPs uptake in hWJ-MSCs. Cells were exposed to HAPs with or without cpz, m $3 c d$, and cytoD, respectively. (A) CLSM images of hWJ-MSCs exposed to HAPs for $2 \mathrm{~h}$. Cells stained for nuclei (blue) and actin (red). HAPs are shown in green color. Scale bars: $50 \mu \mathrm{m}$. (B) Quantification of HAPs uptake after $\mathrm{hWJ}$-MSCs were treated with HAPs for $2 \mathrm{~h}$. ${ }^{* P}<0.01$ versus no inhibitor control, ${ }^{\&} \mathrm{P}<0.05$; ${ }^{\text {\& }} \mathrm{P}<0.0 \mathrm{l}$ versus $\mathrm{m}$-HAP group, ${ }^{\# P<0.01}$ versus np20 group.

Abbreviations: HAPs, hydroxyapatite particles; hWJ-MSCs, human umbilical cord Wharton's jelly-derived mesenchymal stem cells; cpz, chlorpromazine; m $\beta c d$, methyl- $\beta$ cyclodextrin; cytoD, cytochalasin D; CLSM, confocal laser scanning microscopy; np20, hydroxyapatite nanoparticles $20 \mathrm{~nm}$ in diameter; np80, hydroxyapatite nanoparticles $80 \mathrm{~nm}$ in diameter; m-HAP, micro-sized HAP particles; HAP, hydroxyapatite. 
A

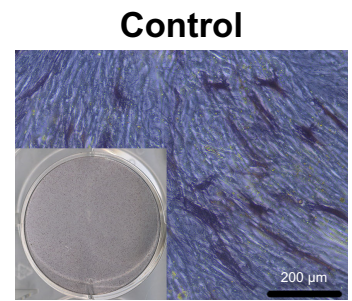

B
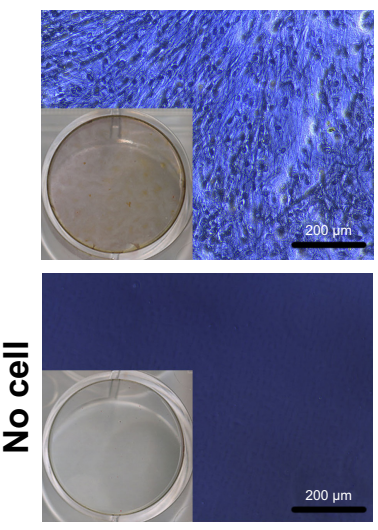

np20
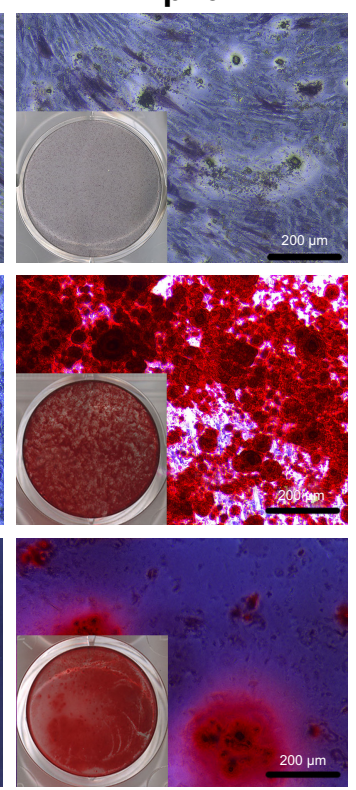

np80
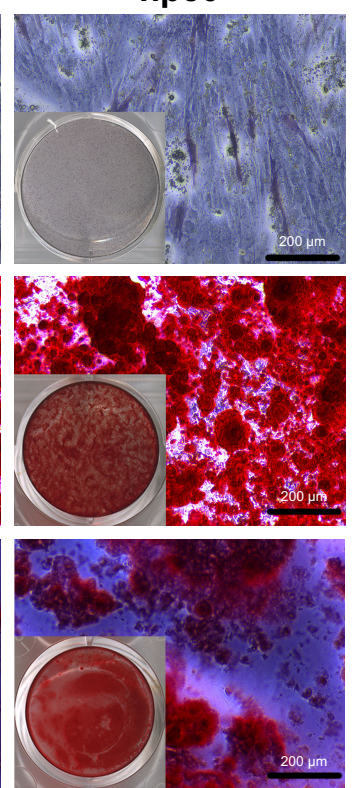

m-HAP
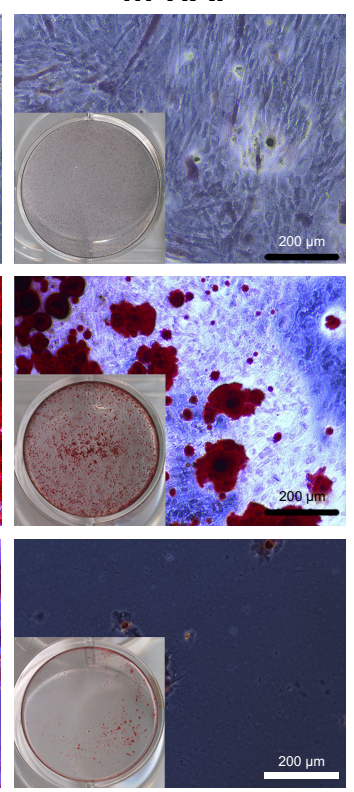

Figure 8 Effects of HAPs on ALP activity and extracellular calcium deposition of hWJ-MSCs. (A) ALP activity of hWJ-MSCs cultured with HAPs for 7 days. (B) The extracellular calcium deposition was visualized by Alizarin Red S staining after cells were cultured with HAPs for I4 days (middle panel). Cells without HAPs treatment were set as the control group (bottom panel).

Abbreviations: HAPs, hydroxyapatite particles; ALP, alkaline phosphatase; hWJ-MSCs, human umbilical cord Wharton's jelly-derived mesenchymal stem cells; np20, hydroxyapatite nanoparticles $20 \mathrm{~nm}$ in diameter; np80, hydroxyapatite nanoparticles $80 \mathrm{~nm}$ in diameter; m-HAP, micro-sized HAP particles; HAP, hydroxyapatite.

To further validate the osteoinductivity of HANPs, we assessed changes in the gene expression level of a panel of osteoblast markers in hWJ-MSCs that were exposed to HAPs. In the HANPs groups, a significant upregulation of $\mathrm{Col} I$ and OCN mRNA on days 14 and 21 was observed compared to m-HAP and control groups. However, the gene expression of Runx-2 and ALP was not elevated by HANPs compared to control (Figure 9).

\section{The activation of JNK and p38-dependent pathways in $\mathrm{hWJ}$-MSCs by HANPs}

To investigate the mechanisms involved in the osteogenic differentiation in hWJ-MSCs treated with HAPs, the regulators in the c-Jun N-terminal kinases (JNK) and p38 signaling pathways were examined. As shown in Figure 10A, p-JNK and p-p38 had increased in the hWJ-MSCs cultured with HAPs, particularly for np20 and np80. However, the expression of total JNK and total p38 remained unchanged. To ascertain whether the activations of JNK and p-p38 were essential for the observed stimulation of hWJ-MSC osteogenic differentiation, we inhibited the JNK and p-p38 pathways using SP600125 and SB203580, respectively, while the hWJ-MSCs were subjected to HANPs induction. As shown in Figure 10B, the extracellular calcium deposition was blocked when cells were cultured in HANPs for 14 days with SP600125 and SB203580.

\section{Discussion}

The unique physical and chemical properties of nanoHAP indicated it as an appealing biomaterial with great potential for hard tissue repair, cancer therapy, and drug delivery. ${ }^{20-22}$ Cytocompatibility is the preliminary issue for exploring the biological behavior of any nanobiomaterial. Remya et al reported that HANPs do not exhibit cytotoxicity up to $800 \mu \mathrm{g} / \mathrm{mL}$ in mouse bone marrow mesenchymal stem cells. ${ }^{23}$ However, previous studies have reported that HANPs inhibited cell viability and induced apoptosis in MC3T3-E1 cells. ${ }^{24}$ Similarly, Zhao et al reported HANPshape-dependent toxicity on BEAS-2B cells. ${ }^{25}$ These results indicated that the cytotoxicity of HANPs largely varied with cell type. According to our findings (Figures 2 and 3), HANPs had no significant cytotoxicity to hWJ-MSCs. This may be attributed to their profile of cellular uptake. Using human umbilical vein endothelial cells, we have previously shown that a large amount of HANPs was taken up during observation. ${ }^{26}$ Comparatively, in the present study, less HANPs were internalized by hWJ-MSCs (Figure 6B). Moreover, HANPs accumulated throughout the interior of electron-dense vacuoles (Figure 4); consequently, at the same dose, there were fewer HANPs containing vacuoles in the cytoplasm. Therefore, HANPs were less likely to influence the organelle function in hWJ-MSCs. On the other hand, HANPs were exclusively localized in the cytoplasmin; thus, 
A

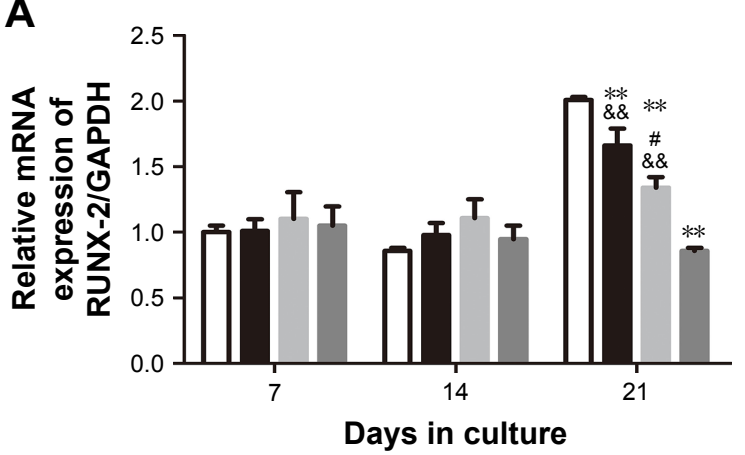

C

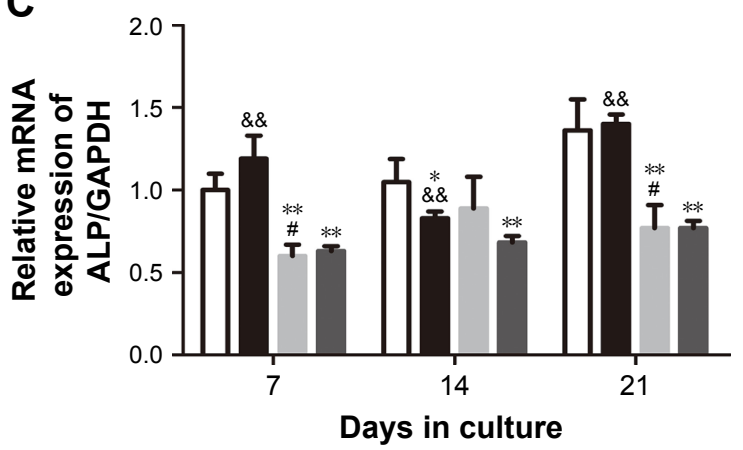

B

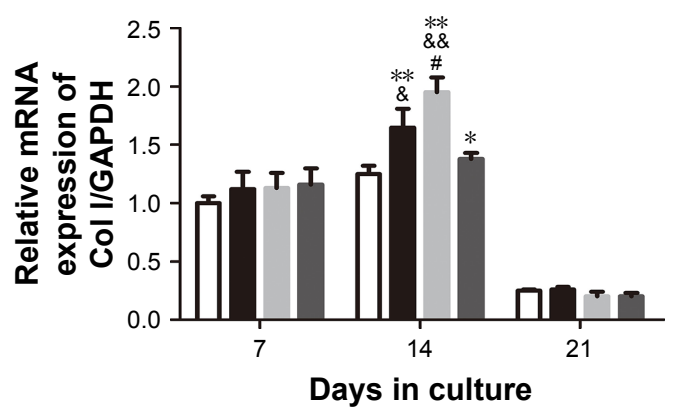

D

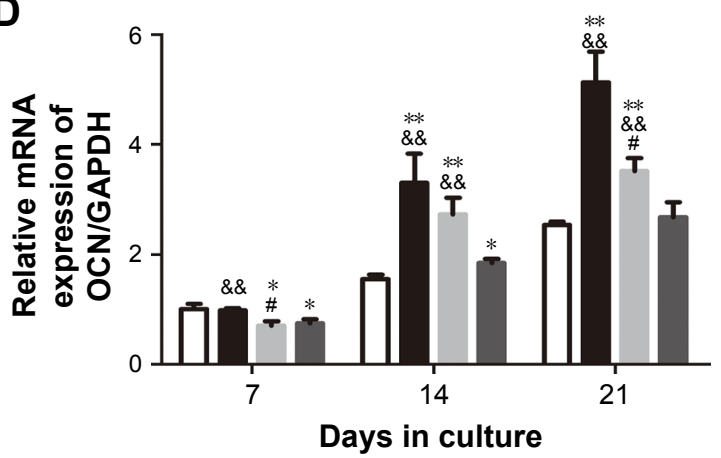

$\square$ Control $\square \mathrm{np20} \square \mathrm{np80} \square \mathrm{m}-\mathrm{HAP}$

Figure 9 Gene expression profile of osteogenic differentiation related gene of hWJ-MSCs cultured with HAPs. (A) RUNX-2; (B) Col I; (C) ALP; (D) OCN. *P $<0.05$; **P $<0.0$ I versus control group, ${ }^{\circledR} P<0.05 ;{ }^{2 \&} P<0.01$ versus $\mathrm{m}-\mathrm{HAP}$ group, ${ }^{\sharp} P<0.01$ versus np20 group. Cells without HAPs treatment were set as the control group. Abbreviations: hWJ-MSCs, human umbilical cord Wharton's jelly-derived mesenchymal stem cells; HAPs, hydroxyapatite particles; ALP, alkaline phosphatase; np20, hydroxyapatite nanoparticles $20 \mathrm{~nm}$ in diameter; np80, hydroxyapatite nanoparticles $80 \mathrm{~nm}$ in diameter; m-HAP, micro-sized HAP particles; HAP, hydroxyapatite.

they exerted negligible adverse effects on genomic DNA and nuclear proteins, which play crucial roles in some key cellular processes. m-HAP has been recognized for its biocompatibility, however, in this study, it triggered up to the most significant decrease in cell viability (Figure 2A). Elegant experiments by Tang et al suggested that the dissolution of NPs may be inhibited when their sizes are similar to a critical value, which is always at a nanoscale level. ${ }^{27}$ Consequently, we presumed that compared to HANPs, too much calcium and phosphate ions from the dissolution of m-HAP will be released into the culture medium, thus suppressing the cell viability of hWJ-MSCs.

Increasing evidence suggests that NP uptake originates from two processes: ${ }^{15}$ firstly, NPs adhere to the cells and interact with cell membrane components, such as lipids and proteins. Then, an energy-dependent uptake is activated, ${ }^{28,29}$ with which the NPs are internalized into the cells and are further delivered to different subcellular locations. ${ }^{30,31}$ In this study, the amount of HAPs adhering to hWJ-MSCs remained substantially constant throughout the observation (Figure 6A), implying that HAP adhesion is a very quick process, and that a steady state can be reached within a few minutes, where the number of HAPs adhering to cells is balanced via desorption. Moreover, we noticed that fewer m-HAP adhered to the cells compared to HANPs, which might be ascribed to the lower energy of their surface. Consistently, the uptake kinetics of m-HAP exhibited a lower initial transient followed by approximately linear uptake (Figure 6B), suggesting HAP adhesion to the cell membrane as an important step in their uptake efficiency. It has been reported that some NPs in the cytoplasm could be trafficked into the nucleus via nuclear pore complexes (NPCs), and correspondingly induce decisive effects on cells. $^{32,33}$ NPs may be translocated into the cell nucleus when their size is close to the diameter of NPCs, which is nearly 20-50 nm and cell-dependent. ${ }^{34}$ Yuan et al confirmed that HANPs with diameters of $25 \mathrm{~nm}$ and $46 \mathrm{~nm}$ were readily trafficked into the nucleus. ${ }^{21}$ However, no HANPs were detected inside the cell nucleus in our study. This may be attributed to the increased size of HANPs due to NPs agglomeration; as HANPs' size increases beyond the diameter of NPCs, their nuclear trafficking may be hindered. Another possibility is associated with the permeability of the nuclear membrane, which could be increased by immature NPCs. ${ }^{35}$ It is clear that the NPCs of the hWJ-MSCs are more mature. 


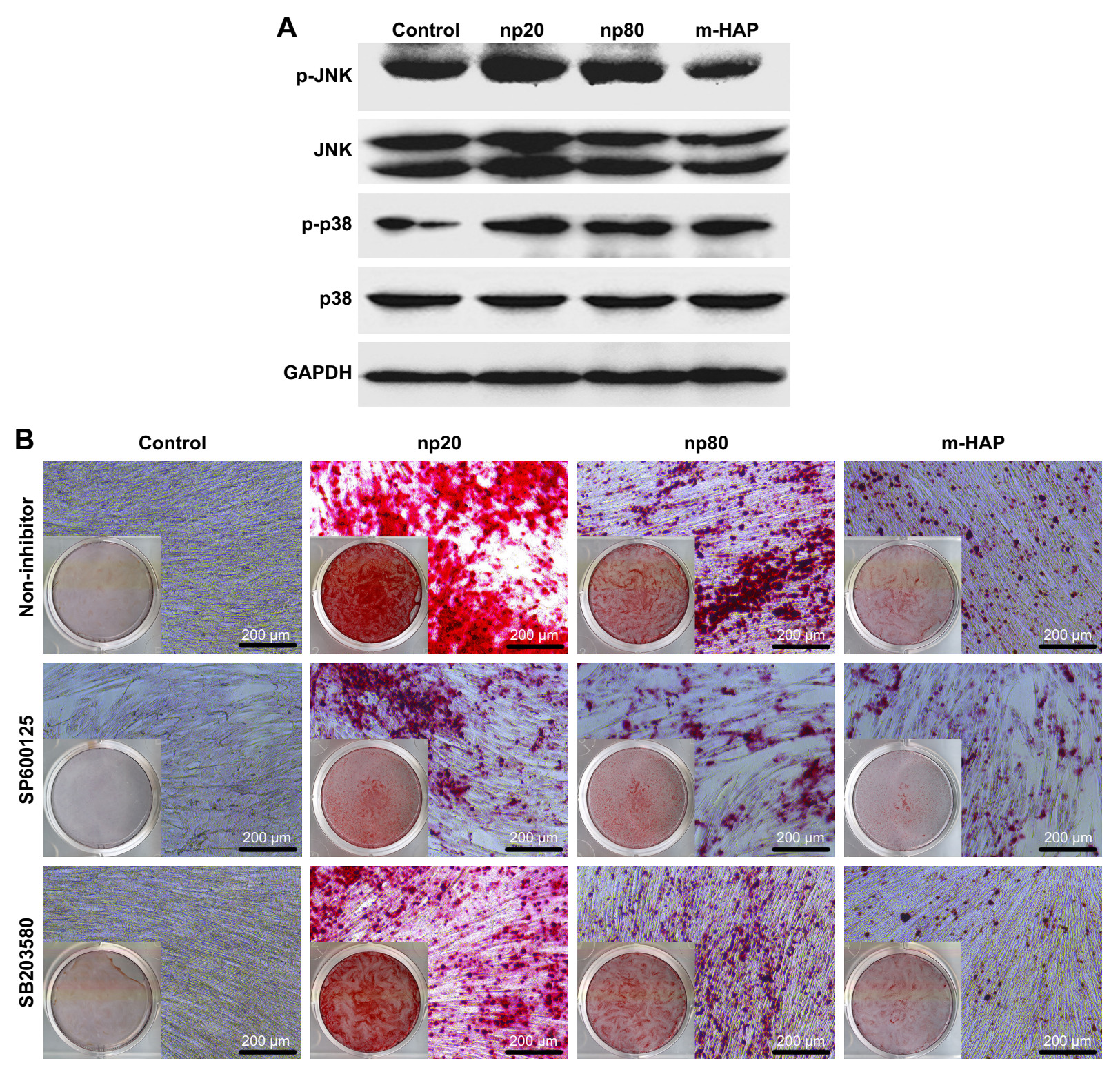

Figure 10 HAPs induce osteogenic differentiation of hWJ-MSCs via activation of JNK and p38 signaling pathways. (A) The expression profile of key proteins in JNK and p38 signaling pathways in hWJ-MSCs cultured with np20, np80, and m-HAP for $24 \mathrm{~h}$. (B) The extracellular calcium deposition in hWJ-MSCs cultured in HAPs for 14 days with or without SP600I 25 and SB203580, respectively. Cells without HAPs treatment were set as the control group.

Abbreviations: HAPs, hydroxyapatite particles; hWJ-MSCs, human umbilical cord Wharton's jelly-derived mesenchymal stem cells; JNK, c-Jun N-terminal kinases; np20, hydroxyapatite nanoparticles $20 \mathrm{~nm}$ in diameter; np80, hydroxyapatite nanoparticles $80 \mathrm{~nm}$ in diameter; m-HAP, micro-sized HAP particles; HAP, hydroxyapatite.

It is furthermore well known that cells have various endocytic routes to uptake material from the extracellular milieu. ${ }^{16,36}$ Particles with sizes above $500 \mathrm{~nm}$ are typically ingested via phagocytosis or macropinocytosis, while internalization of cargo below this size limit often occurs via other diverse endocytic pathways available at the cell surface, such as clathrin-mediated as well as caveolin-mediated endocytosis. ${ }^{37}$ However, although HAP has been extensively investigated in various biomedical applications, the internalization mechanism of HANPs in MSCs still remains unknown. In this study, we have investigated the endocytic processes, focusing on their mechanism of internalization, with the intention of characterizing the endocytosis of HANPs in hWJ-MSCs. It has been previously described that caveolin-mediated endocytosis is mainly detected in several cell types, such as the capillary endothelium, smooth muscle cells, type I alveolar epithelial cells, and fibroblasts. ${ }^{38}$ In our study, the results of microscopy and quantification showed that in the $n p 80$ and $m-H A P$ groups, $m \beta c d$ did not exhibit more effective inhibition compared with cpz (Figure 7). It seems that caveolin-mediated endocytosis was not implicated in the internalization of np80 and m-HAP in hWJ-MSCs. However, all the three endocytosis inhibitors significantly impeded the uptake of np20, and the strength 
of the effect ranged in the order of $\mathrm{m} \beta \mathrm{cd}>\mathrm{cytoD}>\mathrm{cpz}$, suggesting that actin-dependent macropinocytosis as well as clathrin- and caveolin-mediated endocytosis were involved in the internalization of np20. In this context, we presumed that caveolin proteins exist on the cell membranes of hWJ-MSCs; nevertheless, whether HAPs can be taken up through caveolin-mediated endocytosis depends on several factors such as their shape and size. Remarkably, cytoD did not block np80 internalization and cpz only reduced its ingestion to about $70 \%$. This means that for except clathrin-mediated endocytosis, it is highly likely that other important uptake routes are waiting to be found. Previous studies indicated that clathrin could coat vesicles, which have a diameter of 150-200 nm. ${ }^{37}$ However, we found that cpz inhibited the uptake of m-HAP; furthermore, elegant experiments have demonstrated that NPs below $60 \mathrm{~nm}$ could also enter cells via clathrin-mediated endocytosis. ${ }^{16,39}$ In summary, these findings indicate that the size of clathrin-coating vesicles might be outside the range of 150-200 nm. Given the complexity and diversity of uptake routes available in different cells, here, we explored only three main endocytic modes of these pathways. A detailed understanding and elucidation of the uptake mechanisms of HANPs in hWJ-MSCs is complex and will require extensive further work.

Most available data suggested that nano-HAP composite scaffolds have stimulatory ability for osteogenesis. ${ }^{9,40-42}$ It has been accepted that an ideal bone scaffold possesses an appropriate degradation rate that matches osteogenesis in vivo; consequently, nano-HAP from the degradation of bone substitutes would directly interact with some key cells involved in bone repair. In the present study, the impact of HANPs on osteogenic differentiation of hWJ-MSCs was assessed to gain insight into their osteogenic potential. Col I is the protein framework of bone tissue and its expression has widely been used as an indication for osteoblast differentiation in vitro. Most importantly in the current study, in the absence of osteoinductive additives, Col I mRNA was upregulated on day 14 by HANPs compared to m-HAP and control, indicating that HANPs promote osteoblast differentiation and extracellular matrix production. In vitro, osteoblast differentiation consists of an ordered sequence of events, characterized by a temporal expression of genes. Synthesis of the OCN was measured as a marker of the onset of matrix deposition and denotes late stages of osteoblast differentiation. ${ }^{43}$ Interestingly, OCN mRNA levels for cells treated with HANPs were significantly higher on day 14, correlating with the formation of bone nodules (Figures $8 \mathrm{~B}$ and 9). This confirmed that HANPs have the ability to stimulate hWJ-MSCs differentiation toward the osteogenic lineage and thus promote calcium deposition.

The technological advances of artificial biomaterial have made it a promising alternative approach with great potential for bone repair. However, developing bone substitute with a stimulatory ability for osteogenesis remains challenging. So far, the most common approach is to deliver potent osteogenic inductive factors to bone grafting materials. Compared to this approach, biomaterial-based strategies for enhancing osteogenesis offer specific superiority, such as favorable controllability, sustainability, and reduced cost. Since this study has shown that HANPs could induce hWJ-MSC differentiation, it is a central issue to clarify the precise signal transduction mechanism of the stimulation process. It has been documented that mitogen-activated protein kinases (MAPKs; including extracellular signal-regulated kinases, JNK, and p38) are involved in modulating stem cell selfrenewal and differentiation. ${ }^{44-47}$ However, the exact role of MAPKs in the osteogenic differentiation of stem cell still remains a matter of debate. In the present study, it is worth noting that HANPs were able to augment the phosphorylation of JNK and p38, upregulate mRNA levels of Col I and $\mathrm{OCN}$, and stimulate calcium mineral deposition (Figures 8B, 9, and 10A). Moreover, these effects were significantly attenuated by treatment with SP600125 and SB203580, specific JNK and p38 inhibitors, indicating that HANPs might play an important role in the induction of osteogenic differentiation of hWJ-MSCs through JNK and p38 signaling (Figure 10B).

\section{Conclusion}

This study explored the specific biological responses during HANPs' interface with MSCs. Both np20 and np80 had excellent biocompatibility according to the results of cell viability, apoptosis response, and cell cycle. Compared to m-HAP, higher adhesion led to a concomitant increase in HANPs' uptake efficiency. As an important property of NMs, the size of HANPs seemed to have no significant influence on their kinetics of adhesion and uptake; nevertheless, it affected the endocytic routes of HANPs. Our data showed that multiple endocytic pathways including clathrin- and caveolin-mediated endocytosis as well as macropinocytosis operate concurrently in the uptake of np20. By contrast, in addition to clathrin-mediated endocytosis, as-yet-unidentified uptake mechanisms are implicated in the uptake of np80. More remarkably, the in vitro results suggested that HANPs were capable of directing hWJ-MSC differentiation toward the osteogenic lineage through JNK 
and p38 signaling pathways. These findings collectively indicate that nano-HAP could play a beneficial role in bone reconstruction, which might be related to the improved osteogenic differentiation of MSCs.

\section{Acknowledgments}

This study was supported by grants from the National Natural Science Foundation of China (number 81300913), the Natural Science Foundation of the Jiangsu Higher Education Institutions of China (number 13KJB320007), the Natural Science Foundation of Jiangsu Province of China (number BK20161566), and A Project Funded by the Priority Academic Program Development of Jiangsu Higher Education Institutions (number 2014-37).

\section{Disclosure}

The authors report no conflicts of interest in this work.

\section{References}

1. Tay CY, Cai P, Setyawati MI, et al. Nanoparticles strengthen intracellular tension and retard cellular migration. Nano Lett. 2013;14(1):83-88.

2. Setyawati MI, Tay CY, Leong DT. Exploiting cancer's antioxidative weakness through p53 with nanotoxicology. Nanomedicine (Lond). 2014;9(4):369-371.

3. Ribeiro N, Sousa SR, van Blitterswijk CA, Moroni L, Monteiro FJ. A biocomposite of collagen nanofibers and nanohydroxyapatite for bone regeneration. Biofabrication. 2014;6(3):035015.

4. Bral A, Mommaerts MY. In vivo biofunctionalization of titanium patient-specific implants with nano hydroxyapatite and other nano calcium phosphate coatings: a systematic review. J Craniomaxillofac Surg. 2016;44(4):400-412.

5. Abd El-Fattah H, Helmy Y, El-Kholy B, Marie M. In vivo animal histomorphometric study for evaluating biocompatibility and osteointegration of nano-hydroxyapatite as biomaterials in tissue engineering. J Egypt Natl Canc Inst. 2010;22(4):241-250.

6. Zhou H, Lee J. Nanoscale hydroxyapatite particles for bone tissue engineering. Acta Biomater. 2011;7(7):2769-2781.

7. Shi Z, Huang X, Cai Y, Tang R, Yang D. Size effect of hydroxyapatite nanoparticles on proliferation and apoptosis of osteoblast-like cells. Acta Biomater. 2009;5(1):338-345.

8. Zhu W, Wang D, Peng L, et al. An experimental study on the application of radionuclide imaging in repairing bone defects. Artif Cells Nanomed Biotechnol. 2013;41(5):304-308.

9. Hu J, Yang Z, Zhou Y, Liu Y, Li K, Lu H. Porous biphasic calcium phosphate ceramics coated with nano-hydroxyapatite and seeded with mesenchymal stem cells for reconstruction of radius segmental defects in rabbits. J Mater Sci Mater Med. 2015;26(11):257.

10. Santos C, Gomes PS, Duarte JA, et al. Relevance of the sterilizationinduced effects on the properties of different hydroxyapatite nanoparticles and assessment of the osteoblastic cell response. JR Soc Interface. 2012;9(77):3397-3410.

11. Bauer IW, Li SP, Han YC, Yuan L, Yin MZ. Internalization of hydroxyapatite nanoparticles in liver cancer cells. J Mater Sci Mater Med.2008; 19(3):1091-1095.

12. Lock J, Liu H. Nanomaterials enhance osteogenic differentiation of human mesenchymal stem cells similar to a short peptide of BMP-7. Int J Nanomedicine. 2011;6:2769-2777.

13. Hu J, Zhou Y, Huang L, Liu J, Lu H. Effect of nano-hydroxyapatite coating on the osteoinductivity of porous biphasic calcium phosphate ceramics. BMC Musculoskelet Disord. 2014;15:114.
14. Qiao C, Xu W, Zhu W, et al. Human mesenchymal stem cells isolated from the umbilical cord. Cell Biol Int. 2008;32(1):8-15.

15. Lesniak A, Salvati A, Santos-Martinez MJ, Radomski MW, Dawson KA, Åberg C. Nanoparticle adhesion to the cell membrane and its effect on nanoparticle uptake efficiency. $J$ Am Chem Soc. 2013;135(4): 1438-1444.

16. Kuhn DA, Vanhecke D, Michen B, et al. Different endocytotic uptake mechanisms for nanoparticles in epithelial cells and macrophages. Beilstein J Nanotechnol. 2014;5:1625-1636.

17. Li Z, Hüve J, Krampe C, et al. Internalization pathways of anisotropic disc-shaped zeolite L nanocrystals with different surface properties in HeLa cancer cells. Small. 2013;9(9-10):1809-1820.

18. Wang C, Lin K, Chang J, Sun J. The stimulation of osteogenic differentiation of mesenchymal stem cells and vascular endothelial growth factor secretion of endothelial cells by $\beta-\mathrm{CaSiO} 3 / \beta-\mathrm{Ca} 3(\mathrm{PO} 4) 2$ scaffolds. J Biomed Mater Res A. 2013;102(7):2096-2104.

19. Kim EK, Lim S, Park JM, et al. Human mesenchymal stem cell differentiation to the osteogenic or adipogenic lineage is regulated by AMPactivated protein kinase. J Cell Physiol. 2012;227(4):1680-1687.

20. Ning L, Malmström H, Ren YF. Porous collagen-hydroxyapatite scaffolds with mesenchymal stem cells for bone regeneration. J Oral Implantol. 2015;41(1):45-49.

21. Yuan Y, Liu C, Qian J, Wang J, Zhang Y. Size-mediated cytotoxicity and apoptosis of hydroxyapatite nanoparticles in human hepatoma HepG2 cells. Biomaterials. 2010;31(4):730-740.

22. Do TN, Lee WH, Loo CY, Zavgorodniy AV, Rohanizadeh R. Hydroxyapatite nanoparticles as vectors for gene delivery. Ther Deliv. 2012; 3(5):623-632.

23. Remya NS, Syama S, Gayathri V, Varma HK, Mohanan PV. An in vitro study on the interaction of hydroxyapatite nanoparticles and bone marrow mesenchymal stem cells for assessing the toxicological behaviour. Colloids Surf B Biointerfaces. 2014;117:389-397.

24. Wang L, Zhou G, Liu H, et al. Nano-hydroxyapatite particles induce apoptosis on MC3T3-E1 cells and tissue cells in SD rats. Nanoscale. 2012;4(9):2894-2899.

25. Zhao X, Ng S, Heng BC, et al. Cytotoxicity of hydroxyapatite nanoparticles is shape and cell dependent. Arch Toxicol. 2013;87(6):1037-1052.

26. Shi X, Zhou K, Huang F, Wang C. Interaction of hydroxyapatite nanoparticles with endothelial cells: internalization and inhibition of angiogenesis in vitro through the PI3K/Akt pathway. Int J Nanomedicine. 2017; 12:5781-5795

27. Tang R, Wang L, Orme CA, Bonstein T, Bush PJ, Nancollas GH. Dissolution at the nanoscale: self-preservation of biominerals. Angew Chem Int Ed Engl. 2004;43(20):2697-2701.

28. Dausend J, Musyanovych A, Dass M, et al. Uptake mechanism of oppositely charged fluorescent nanoparticles in HeLa cells. Macromol Biosci. 2008;8(12):1135-1143.

29. Chithrani BD, Ghazani AA, Chan WC. Determining the size and shape dependence of gold nanoparticle uptake into mammalian cells Nano Lett. 2006;6(4):662-668.

30. Salvati A, Aberg C, dos Santos T, et al. Experimental and theoretical comparison of intracellular import of polymeric nanoparticles and small molecules: toward models of uptake kinetics. Nanomedicine. 2011;7(6): $818-826$.

31. Shapero K, Fenaroli F, Lynch I, Cottell DC, Salvati A, Dawson KA. Time and space resolved uptake study of silica nanoparticles by human cells. Mol Biosyst. 2011;7(2):371-378.

32. Fahrenkrog B, Aebi U. The nuclear pore complex: nucleocytoplasmic transport and beyond. Nat Rev Mol Cell Biol. 2003;4(10):757-766.

33. Shahin V. Cellular transport: gatekeepers of the nucleus. Nat Nanotechnol. 2016;11(8):658-659.

34. Wente SR. Gatekeepers of the nucleus. Science. 2000;288(5470): 1374-1377.

35. Boulares AH, Yakovlev AG, Ivanova V, et al. Role of poly(ADP-ribose) polymerase (PARP) cleavage in apoptosis. Caspase 3-resistant PARP mutant increases rates of apoptosis in transfected cells. $J$ Biol Chem. 1999;274(33):22932-22940. 
36. Brandenberger C, Mühlfeld C, Ali Z, et al. Quantitative evaluation of cellular uptake and trafficking of plain and polyethylene glycol-coated gold nanoparticles. Small. 2010;6(15):1669-1678.

37. Kumari S, Mg S, Mayor S. Endocytosis unplugged: multiple ways to enter the cell. Cell Res. 2010;20(3):256-275.

38. Conner SD, Schmid SL. Regulated portals of entry into the cell. Nature. 2003;422(6927):37-44.

39. Xia Y, You P, Xu F, Liu J, Xing F. Novel functionalized selenium nanoparticles for enhanced anti-hepatocarcinoma activity in vitro. Nanoscale Res Lett. 2015;10(1):1051.

40. Luo Y, Lode A, Wu C, Chang J, Gelinsky M. Alginate/nanohydroxyapatite scaffolds with designed core/shell structures fabricated by $3 \mathrm{D}$ plotting and in situ mineralization for bone tissue engineering. ACS Appl Mater Interfaces. 2015;7(12):6541-6549.

41. Kim HL, Jung GY, Yoon JH, et al. Preparation and characterization of nano-sized hydroxyapatite/alginate/chitosan composite scaffolds for bone tissue engineering. Mater Sci Eng C Mater Biol Appl. 2015;54: 20-25.

42. Sadat-Shojai M, Khorasani MT, Jamshidi A. 3-Dimensional cell-laden nano-hydroxyapatite/protein hydrogels for bone regeneration applications. Mater Sci Eng C Mater Biol Appl. 2015;49:835-843.
43. Tsigkou O, Jones JR, Polak JM, Stevens MM. Differentiation of fetal osteoblasts and formation of mineralized bone nodules by $45 \mathrm{~S} 5 \mathrm{Bio}-$ glass conditioned medium in the absence of osteogenic supplements. Biomaterials. 2009;30(21):3542-3550.

44. Wang P, Wang Y, Tang W, et al. Bone morphogenetic protein-9 enhances osteogenic differentiation of human periodontal ligament stem cells via the JNK pathway. PLoS One. 2017;12(1):e0169123.

45. Fuchigami S, Nakamura T, Furue K, Sena K, Shinohara Y, Noguchi K. Recombinant human bone morphogenetic protein-9 potently induces osteogenic differentiation of human periodontal ligament fibroblasts. Eur J Oral Sci. 2016;124(2):151-157.

46. Wu Y, Xia L, Zhou Y, Xu Y, Jiang X. Icariin induces osteogenic differentiation of bone mesenchymal stem cells in a MAPK-dependent manner. Cell Prolif. 2015;48(3):375-384

47. Gallea S, Lallemand F, Atfi A, et al. Activation of mitogen-activated protein kinase cascades is involved in regulation of bone morphogenetic protein-2-induced osteoblast differentiation in pluripotent $\mathrm{C} 2 \mathrm{C} 12$ cells. Bone. 2001;28(5):491-498.
International Journal of Nanomedicine

\section{Publish your work in this journal}

The International Journal of Nanomedicine is an international, peerreviewed journal focusing on the application of nanotechnology in diagnostics, therapeutics, and drug delivery systems throughout the biomedical field. This journal is indexed on PubMed Central, MedLine, CAS, SciSearch $®$, Current Contents ${ }^{\circledR} /$ Clinical Medicine,

\section{Dovepress}

Journal Citation Reports/Science Edition, EMBase, Scopus and the Elsevier Bibliographic databases. The manuscript management system is completely online and includes a very quick and fair peer-review system, which is all easy to use. Visit http://www.dovepress.com/ testimonials.php to read real quotes from published authors. 